\title{
Fibrina rica em plaquetas e piezocirúrgico em comparação com instrumento rotatório convencional e coágulo em cirurgia de terceiros molares inferiores impactados: relato de caso
}

\section{Platelet-rich fibrin and piezo surgical unit in comparison to conventional rotational instrument and clot in impacted lower third molar surgery: a case report}

Alana Lemos da Silveira

Alana Lemos da Silveira** Gabriel Rodrigues Oliveira***

Rafael Manfro****

Fernando Malmann ${ }^{* * * *}$

\section{Resumo}

Objetivo: avaliar os resultados pós-operatórios em cirurgias de terceiros molares inferiores impactados com um desenho split mouth. Em um dos lados, foi utilizada fibrina rica em plaquetas (PRF) e piezocirúrgico, no lado oposto, foi realizada osteotomia rotatória convencional e coágulo. Foram avaliados a relação entre as duas técnicas de osteotomia, o tempo cirúrgico e a gravidade das sequelas no pós-operatório, incluindo dor, edema, trismo e reparo. Relato de caso: este trabalho apresenta um relato de caso clínico como projeto-piloto de uma série de casos clínicos. Os terceiros molares inferiores indicados para extração, foram pareados pela classificação de Pell e Gregory, sendo divididos em: grupo A (hemiarco direito), utilizando o piezocirúrgico e PRF, e grupo $B$ (hemiarco esquerdo), seguindo os padrões tradicionais com brocas rotatórias e coágulo. As exodontias foram realizadas em um único momento pelo mesmo cirurgião-dentista. O piezocirúrgico, comparado à técnica convencional, demonstrou menor dor pós-operatória pela escala de VAS, além de diminuir o número de analgésicos consumidos e reduzir significativamente o trismo após 72 horas da cirurgia. Em contrapartida, o seu tempo cirúrgico foi superior ao da técnica convencional. Considerações finais: conclui-se que o uso de piezocirúrgico e PRF obteve melhores resultados nas condições pós-operatórias com relação a dor, edema e reparo. Porém, o tempo cirúrgico foi maior que a osteotomia convencional e coágulo. Sendo assim, o presente estudo mostrou que a piezocirurgia e a utilização combinada de PRF têm efeitos positivos nos resultados pós-operatórios, após a exodontia de terceiro molar impactado.

Palavras-chave: Osteotomia. Cirurgia menor. Dente do siso. Exodontia.

\section{Introdução}

A inclusão dentária pode acometer qualquer elemento dentário, porém é mais frequente nos terceiros molares. Por serem os últimos dentes a irromperem na cavidade oral, frequentemente, apresentam-se impactados. Essa inclusão acontece pela falta de espaço, posição do segundo molar, topografia óssea,

\footnotetext{
Professor do Curso de Odontologia da Faculdade Meridional (Imed), Passo Fundo, RS. Acadêmica do Curso de Odontologia da Imed, Passo Fundo, RS.

Acadêmico do curso de especialização em Implantodontia lodontus, Passo Fundo, RS.

**** Professor do Curso de Cirurgia Avançada em Implantodontia/Implantoexcelência, Florianópolis, SC.

Professor do curso de especialização em Implantodontia lodontus, Passo Fundo, RS.
} 
hereditariedade, tendência evolutiva, alterações patológicas, traumatismos e alterações sistêmicas ${ }^{1}$.

Terceiros molares impactados são comumente encontrados na clínica odontológica, e a remoção desses elementos é indicada, em época adequada, uma vez que pode ocasionar riscos futuros ao paciente, como periocoronarites, dor local, abcessos odontogênicos, trismo, cáries distais, reabsorção radicular, cistos e tumores. Mesmo com a ausência de sintomatologia, esses elementos são normalmente extraídos com intuito de reduzir os sintomas clínicos mencionados. Essa cirurgia, assim como qualquer outra, pode resultar em acidentes e complicações, como hemorragias, dor, edema e trismo ${ }^{2}$.

Essas intercorrências são, em geral, ocasionadas pelo difícil acesso, sendo necessário, na maioria dos casos, a realização de osteotomia, que consiste no seccionamento ou corte cirúrgico do osso, realizado, tradicionalmente, com instrumento rotatório convencional, sendo uma técnica invasiva, que pode gerar acidentes durante a exodontia e desconforto após a sua realização. A extração de dentes impactados pode comprometer os aspectos físicos e psicológicos do paciente, pelo desconforto no pós-operatório. Diferentes estratégias são adotadas para reduzir essas complicações, incluindo a mudança de técnica da osteotomia ${ }^{3-4}$.

Tendo em vista o que foi apresentado, a técnica realizada com uso do piezoelétrico vem sendo empregada, especialmente na odontologia, para gerar menos danos ao paciente. Autores relatam que o dispositivo piezoelétrico utiliza uma frequência ultrassônica, permitindo o corte seguro e preciso do tecido duro, sem provocar danos em plexos nervosos, vasos e tecidos moles ${ }^{5}$.

A piezocirurgia tem características terapêuticas nas osteotomias, como cortes extremamente precisos, seletivos, milimétricos e campo cirúrgico mais seguro. A piezoeletricidade utiliza frequências ultrassônicas que fazem vibrar as pontas especialmente desenhadas para osteotomia. As pontas do instrumento oscilam, permitindo uma osteotomia efetiva, com mínima ou nenhuma injúria a tecidos moles adjacentes, membranas e tecidos nervosos ${ }^{6}$.

O objetivo deste estudo é observar os resultados pós-operatórios em terceiros molares inferiores impactados, que foram tratados usando fibrina rica em plaquetas (PRF) associada ao piezocirúrgico ou a osteotomia rotatória convencional e coágulo, comparando a piezocirurgia com técnicas de osteotomia rotativa, em relação ao tempo de cirurgia e à gravidade das condições pós-operatórias, incluindo dor, edema, trismo e reparo no estudo-piloto.

\section{Relato de caso}

A cirurgia foi realizada em uma paciente do gênero feminino com 21 anos, classificada sistematicamente como ASA I, com indicação de exodontia dos terceiros molares inferiores. $\mathrm{O}$ hemiarco inferior direito foi classificado como grupo $\mathrm{A}$, em que a cirurgia foi realizada com piezocirúrgico e $\mathrm{PRF}$, já o hemiarco inferior esquerdo foi classificado como grupo B, em que a cirurgia foi realizada seguindo os padrões tradicionais, com brocas rotatórias e coágulo. As cirurgias foram realizadas em um único momento, visando estabelecer um comparativo mais fidedigno de parâmetro de dor, edema e reparo do paciente.

Durante a fase pré-operatória, foram avaliados parâmetros como abertura de boca e dimensão facial (edema) (Figuras 1 e 2). A abertura de boca foi mensurada com régua própria e as dimensões faciais com fio de linho, usando a comissura labial e o tragus auditivo como referência, contornando a face do paciente bilateralmente, após, o fio foi esticado e, com auxílio de uma régua, obteve-se a medida em centímetros (cm). No pós-operatório, no mesmo dia, foi avaliada a queixa principal descrita pelo paciente e a quantidade de analgésicos ingeridos. Em seguida, todos os parâmetros, incluindo dor, edema, trismo e número de analgésicos, foram coletados e avaliados, nos primeiros três dias e no sétimo dia após a cirurgia, junto com a aplicação da escala analógica visual (VAS). O estudo também verificou o tempo cirúrgico de cada técnica utilizada.

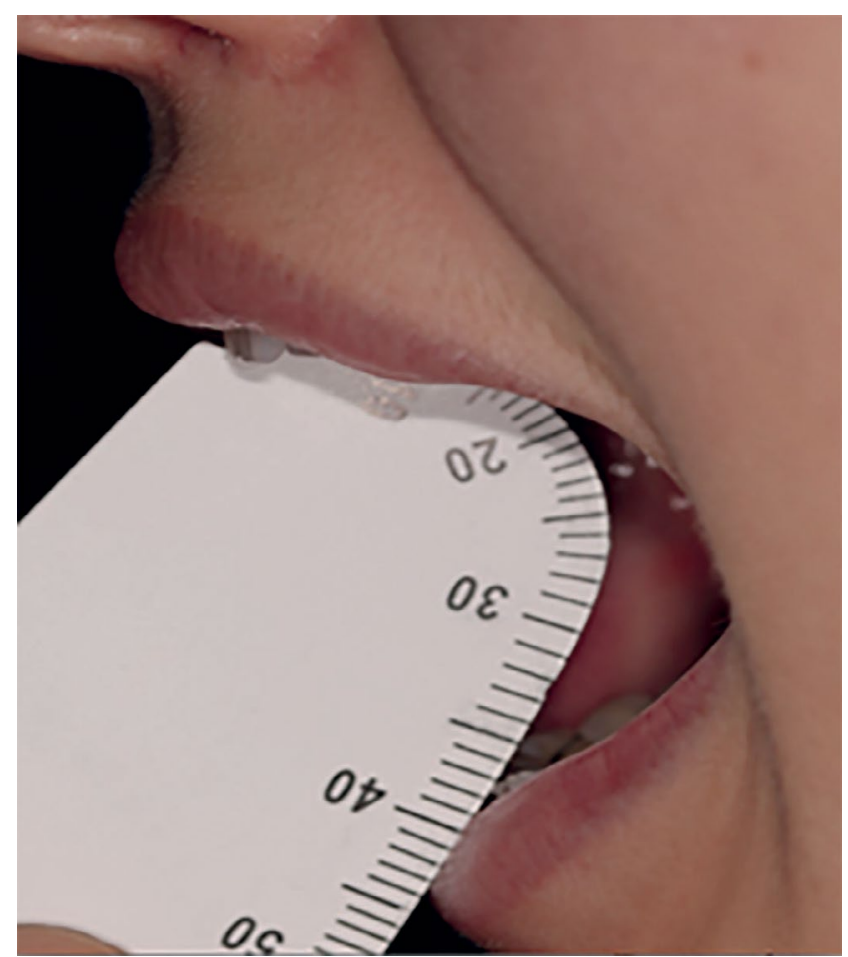

Figura 1 - Medida da abertura bucal inicial

Fonte: dos autores. 


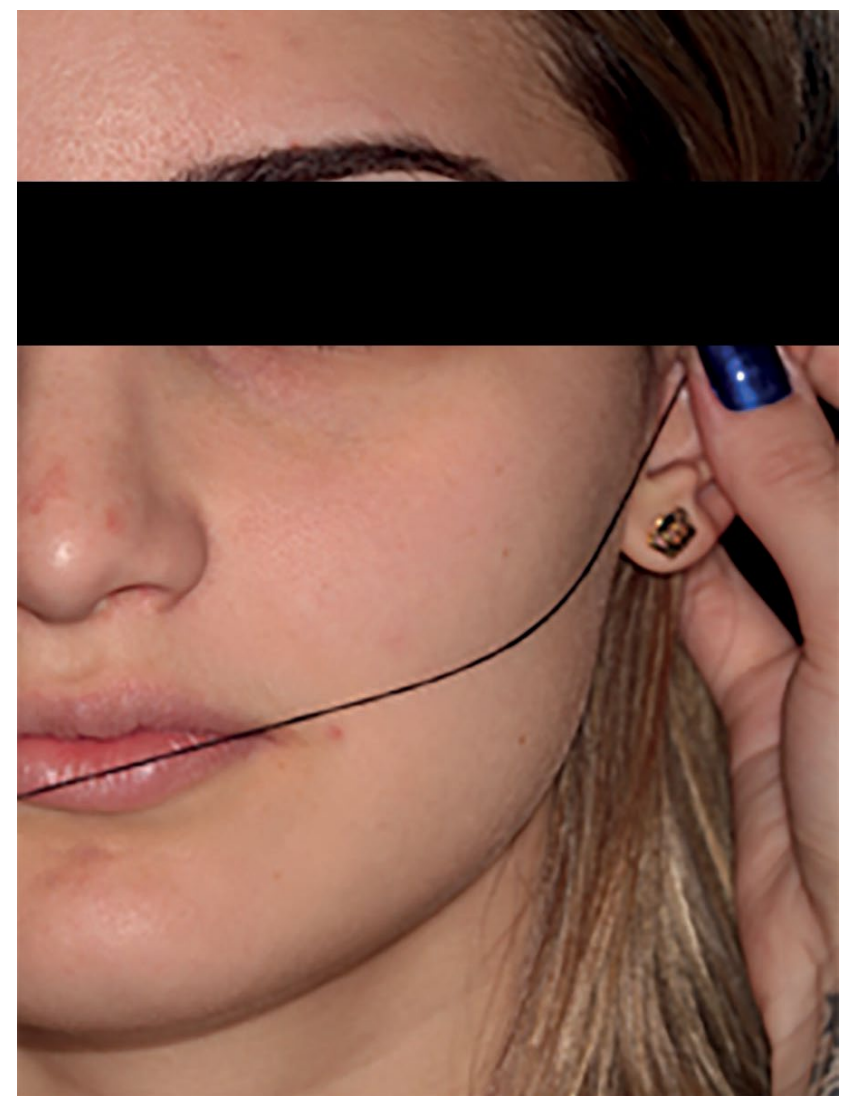

Figura 2 - Mensuração inicial

Fonte: dos autores.

\section{Critérios de inclusão e exclusão}

Os critérios de seleção para o estudo foram: (a) presença bilateral de terceiros molares inferiores, orientados simetricamente, com indicação profiláti-

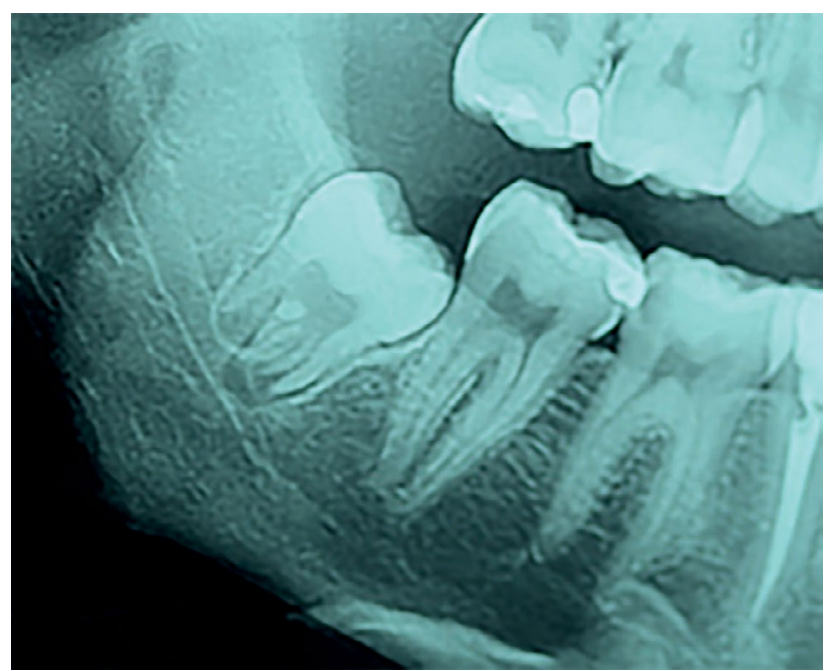

Figura 3 - Visão panorâmica do dente 48

Fonte: dos autores.

O protocolo terapêutico pré-operatório foi de $1 \mathrm{~g}$ de Cefadroxil e $8 \mathrm{mg}$ de Dexametasona, uma hora antes do procedimento cirúrgico, não sendo utiliza- ca de remoção cirúrgica; (b) ausência de discrasias sistêmicas; (c) sem o uso de opioide crônico; (d) idade maior de 18 anos; (e) não fumante e não etilista; (f) não estar grávida; e (g) qualquer alergia à penicilina ou a outras drogas usadas durante a terapia pós-operatória padronizada. Os doentes que tomaram antibióticos para uma infecção atual ou que tiveram quadro de pericoronarite aguda ou doença periodontal grave na data da operação foram excluídos.

De acordo com os critérios mencionados, foi incluído um paciente como estudo de caso, com terceiros molares inferiores bilateralmente e impactados. Além disso, no caso selecionado, os lados apresentavam condições semelhantes de cirurgia com necessidade de osteotomia e odontosecção, bem como dificuldade moderada (classe I, nível C) segundo a classificação de Pell e Gregory.

\section{Fase pré-operatória}

$\mathrm{Na}$ etapa pré-operatória, foi realizada anamnese e foram dadas orientações sobre os riscos inerentes a procedimento cirúrgico, complicações, chance de sucesso, morbidade e demais informações correspondentes. Inicialmente, isso foi realizado de forma verbal, depois, por meio do termo de consentimento livre e esclarecido do cirurgião e da pesquisa.

Para melhor avaliação do caso, foram solicitadas radiografia panorâmica (Figuras 3 e 4) e tomografia computadorizada de feixe cônico, devido à posição do dente e à relação com nervo alveolar inferior (Figura 5 e 6). Exames laboratoriais de rotina, como hemograma, coagulograma, plaquetas e glicemia em jejum, foram solicitados para avaliar a situação sistêmica do paciente.

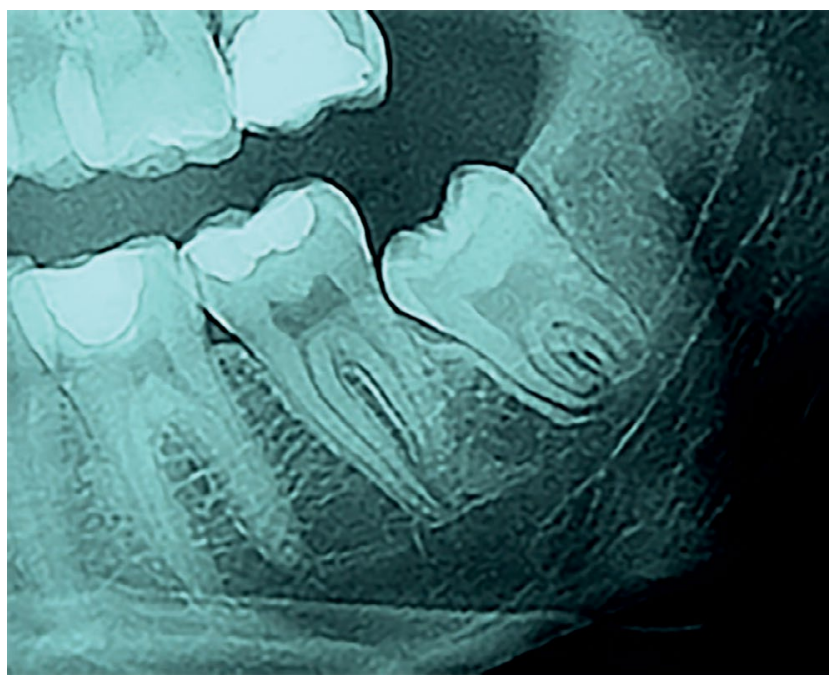

Figura 4 - Visão panorâmica do dente 38

Fonte: dos autores.

do nenhum tipo de sedação, para não aumentar o limiar de dor ou gerar analgesia falsa, podendo ocasionar viés durante a análise dos dados. 


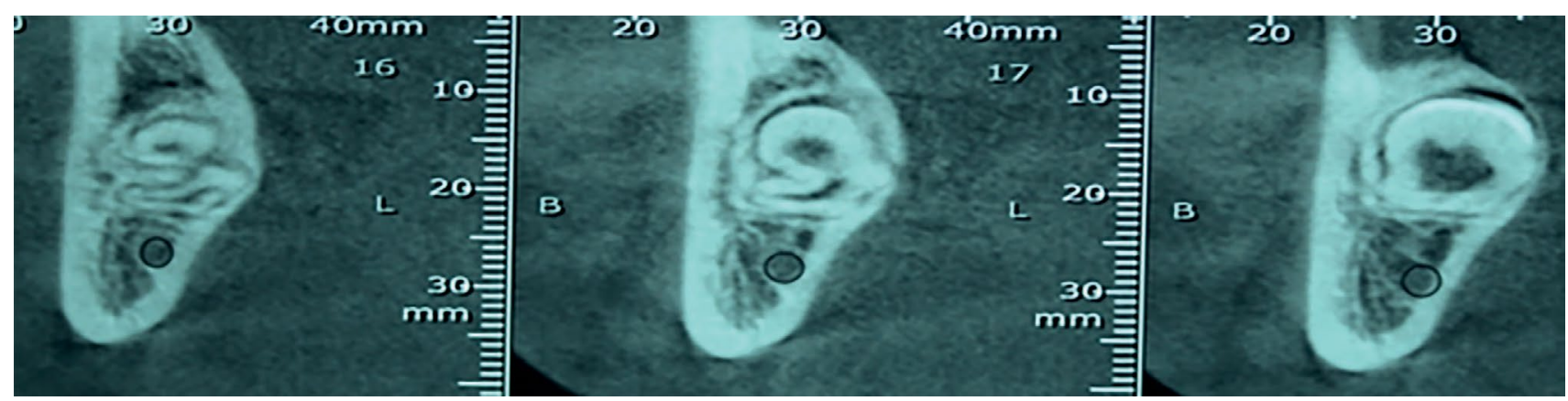

Figura 5 - Tomografia computadorizada evidenciado relação do NAl e dente 48 Fonte: dos autores.
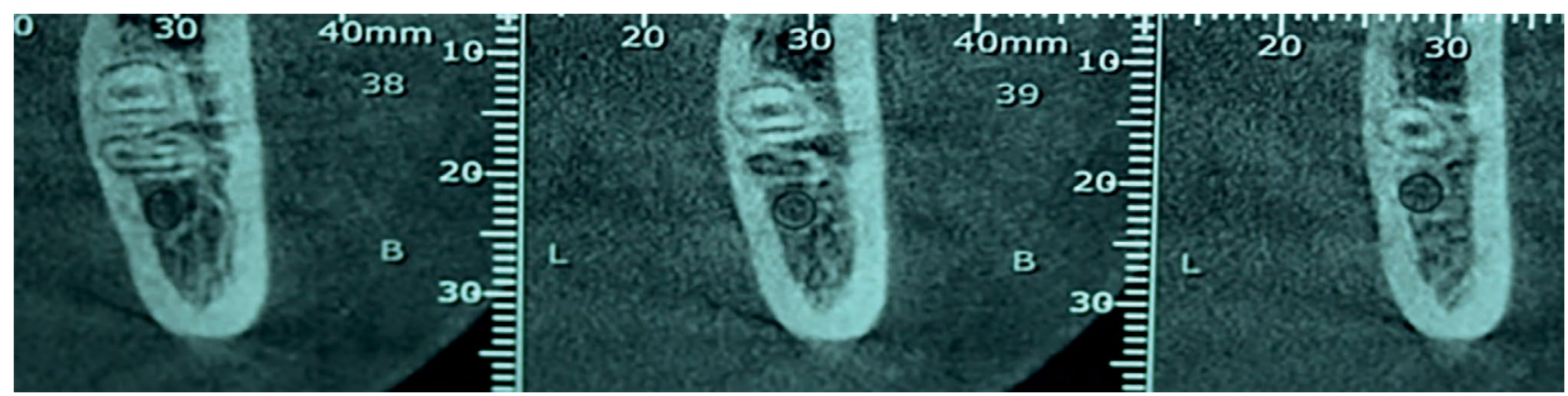

Figura 6 - Tomografia computadorizada evidenciando relação do NAl e dente 38

Fonte: dos autores.

\section{Fase cirúrgica}

O caso foi dividido em dois grupos principais de acordo com o hemiarco mandibular. Para o grupo A (hemiarco direito), a cirurgia utilizou piezocirúrgico e PRF; para o grupo B (hemiarco esquerdo), a cirurgia seguiu os padrões tradicionais, com brocas rotatórias e coágulo. As cirurgias foram realizadas em um único momento, visando estabelecer um comparativo mais fidedigno de parâmetro de dor, edema e reparo do paciente.

Inicialmente, foram realizadas antissepsias intra e extraoral, assim como montagem de campos cirúrgicos para minimizar o risco de contaminação local. O procedimento cirúrgico foi iniciado pelo elemento 48 (grupo A). Foi administrada anestesia com Articaína 4\% com 1:100.000 de Epinefrina

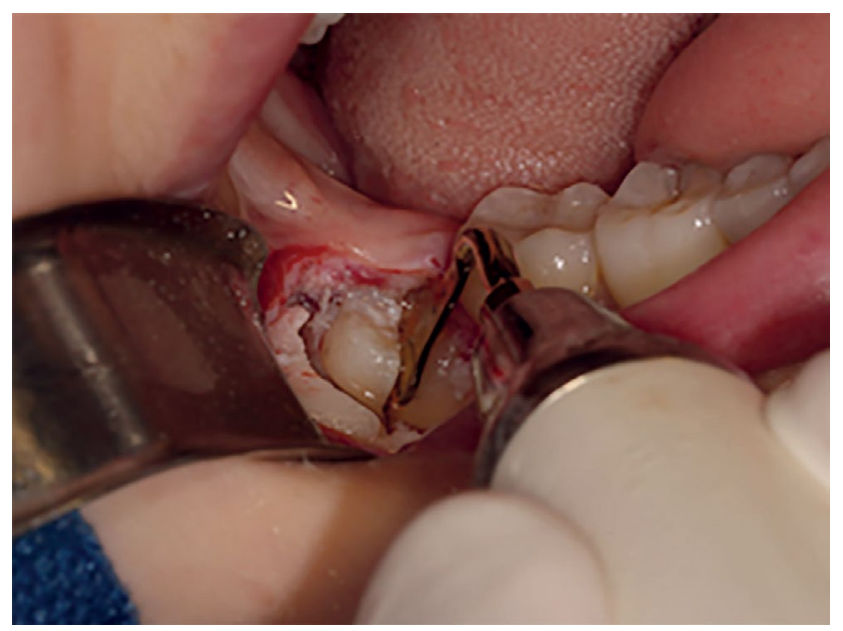

Figura 7 - Osteotomia com Piezo

Fonte: dos autores.
(DFL), por meio de bloqueio dos nervos alveolar inferior, bucal e lingual do lado direito, com total de 2 tubetes anestésicos de 1,8 ml cada.

Após cinco minutos, para efeito do anestésico, deu-se início ao procedimento cirúrgico com incisão supracrestal deslocada para vestibular, com intrasulcular e alívio no 47, descolamento total do retalho, osteotomia e odontosecção com o piezocirúrgico (Figuras 7 e 8) (Woodpecker Dental Ultrasonic), luxação do dente e posterior remoção do elemento dental com alavanca apical reta. No mesmo momento em que a osteotomia e a odontosecção eram realizadas, foi coletado sangue do paciente para confecção do PRF. Após realizar a limpeza da cavidade, o PRF foi inserido no interior do alvéolo em forma de membranas sobrepostas, o retalho foi reposicionado e suturado com fio Mono Nylon 5-0 (Ethicon).

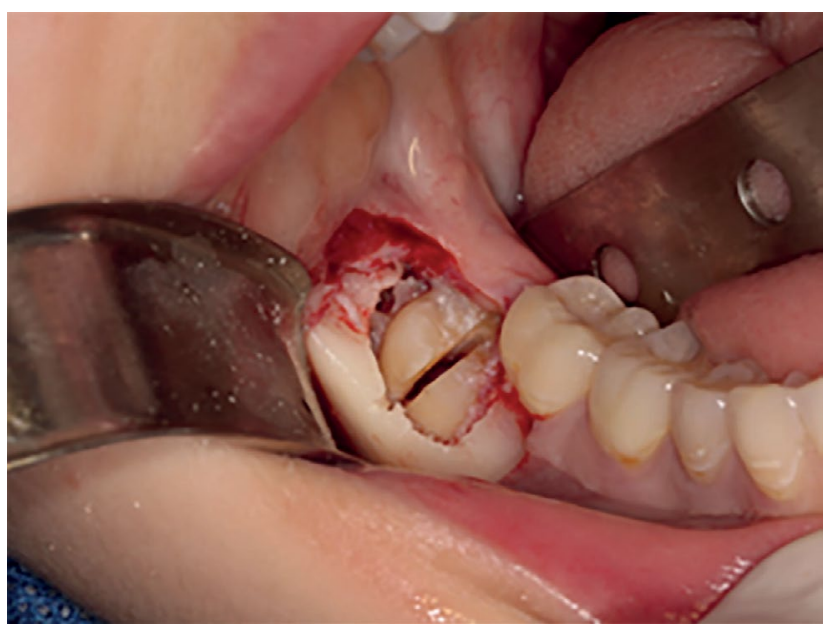

Figura 8 - Odontosecção com Piezo

Fonte: dos autores. 
O PRF foi preparado por meio de coleta de sangue com sistema de coleta a vácuo, utilizando um tubo sem aditivo de anticoagulantes com capacidade para $10 \mathrm{ml}$ de sangue, que imediatamente foi centrifugado em centrífuga (Biotron-MAG M415P)

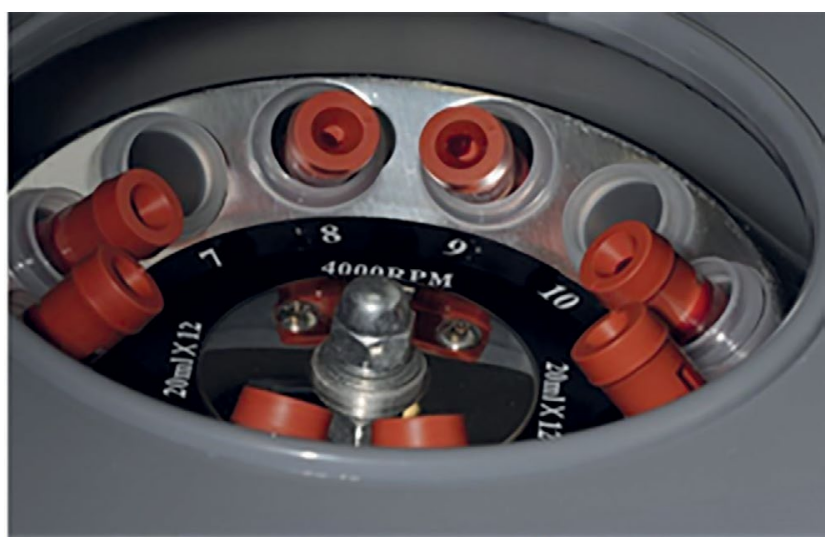

Figura 9 - Centrifugação para confecção do PRF

Fonte: dos autores.

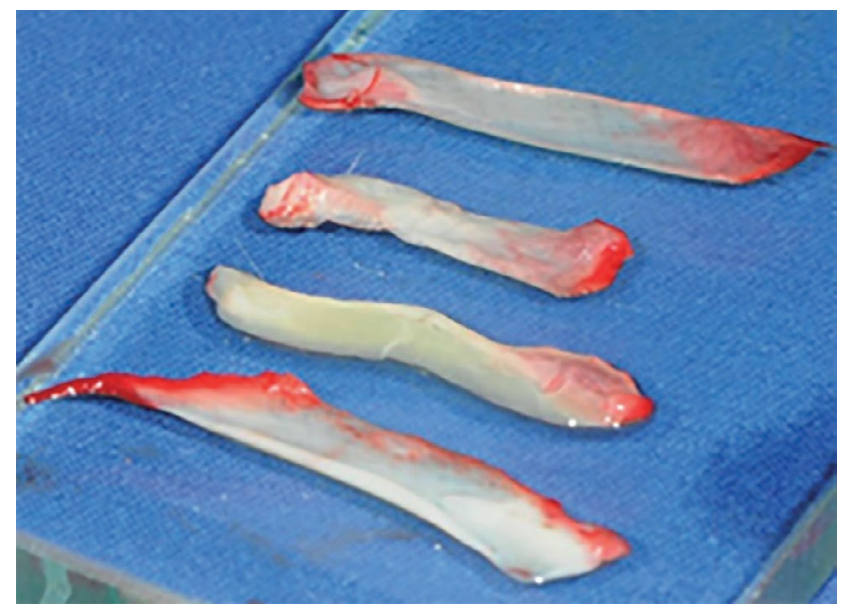

Figura 11 - PRF em formato de membrana

Fonte: dos autores.

Após o término da cirurgia do dente incluso 48, com o tempo cronometrado, iniciou-se o procedimento cirúrgico de remoção do dente 38 (grupo B). A técnica anestésica assim como o tipo de anestésico e a quantidade de tubetes seguiu o mesmo protocolo utilizado no lado direito.

Após cinco minutos para efeito do anestésico, iniciou-se o procedimento cirúrgico com incisão su- a 3.000 rpm durante 10 minutos (aproximadamente $400 \mathrm{~g})^{7}$, a fibrina rica em plaquetas formada foi prensada entre duas placas de vidro e utilizada em forma de membrana preenchendo o interior do alvéolo no grupo $\mathrm{A}$ (Figuras 9 a 12).

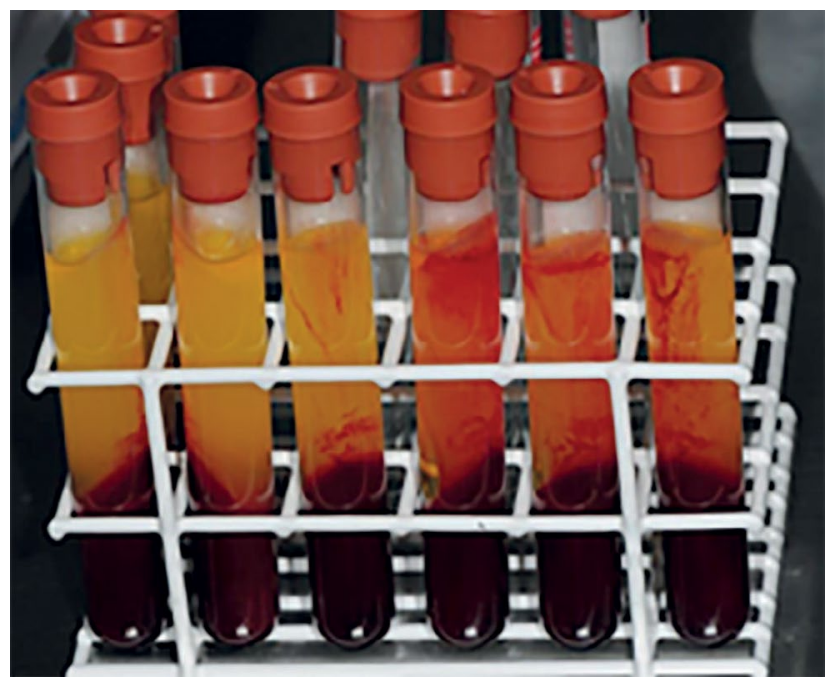

Figura 10 - PRF após centrifugação

Fonte: dos autores.

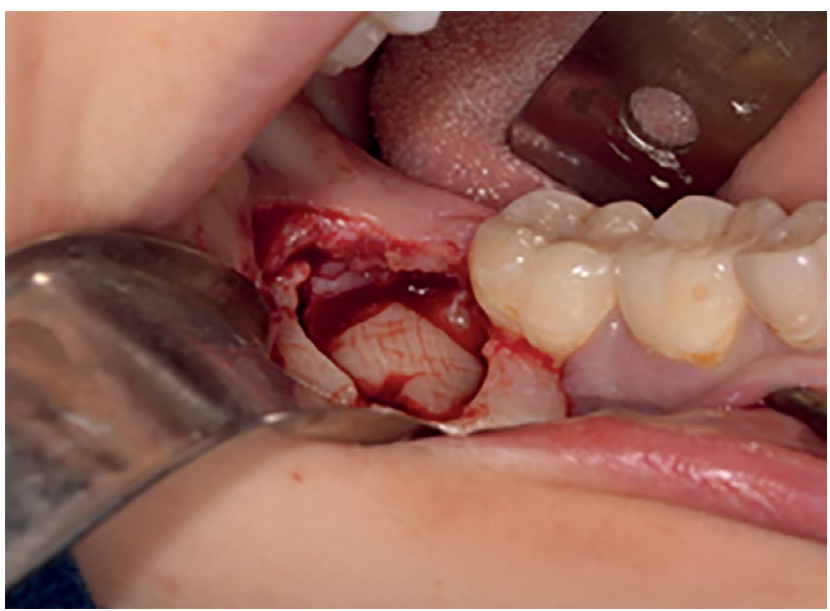

Figura 12 - PRF preenchendo alvéolo

Fonte: dos autores.

pracrestal deslocada para vestibular, com intrasulcular e alívio no 37. A partir do descolamento total do retalho, iniciou-se a osteotomia e odontosecção com broca H33L (Komet) (Figuras 13 e 14) em peça reta com posterior luxação do dente e remoção do elemento com alavanca apical reta. Após o toalete do alvéolo, foi aguardada a formação do coágulo e o retalho foi suturado com fio Mono Nylon 5-0 (Figuras 15 e 16). 


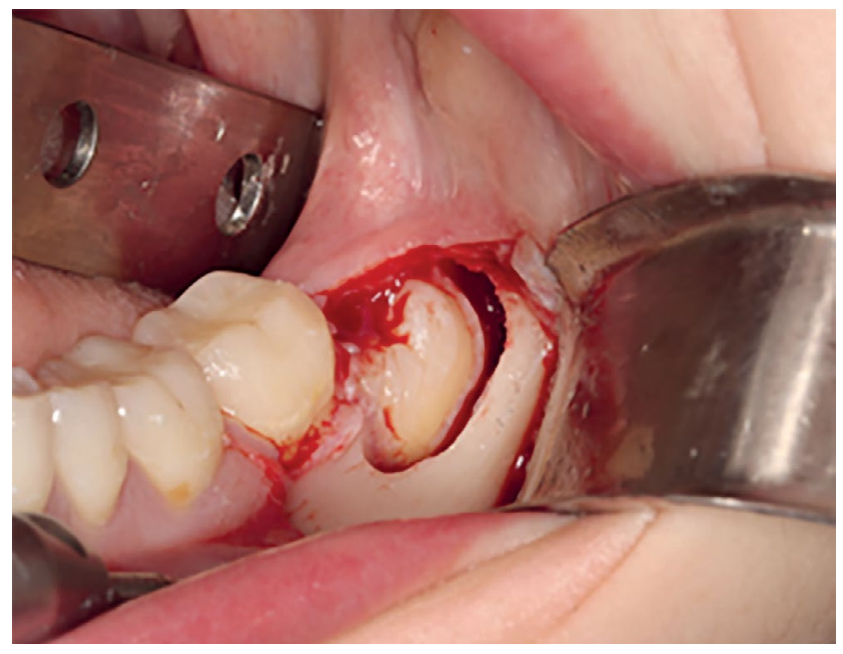

Figura 13 - Osteotomia com broca H33L

Fonte: dos autores.

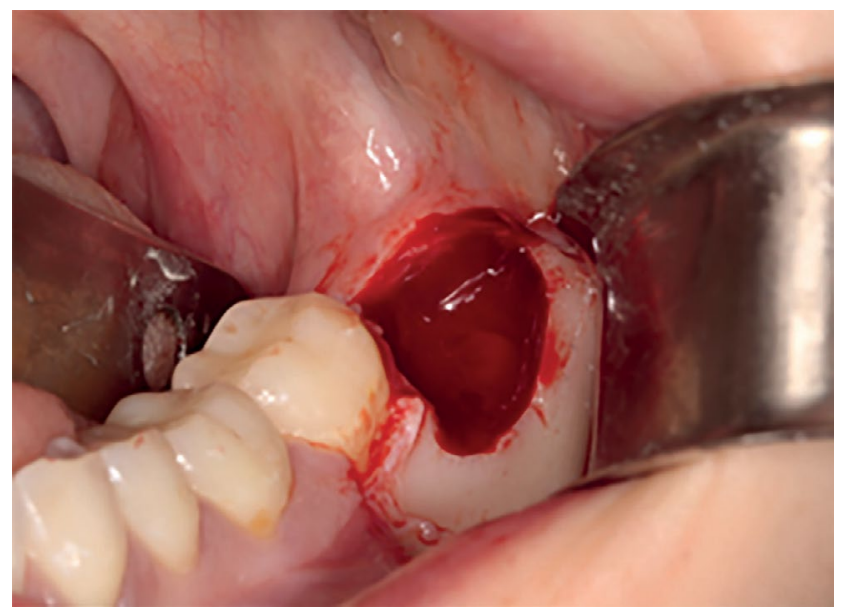

Figura 15 - Coágulo formado

Fonte: dos autores.

Após o término do procedimento, a paciente recebeu todas as orientações pós-operatórias nas formas verbal e escrita e foi medicada com Nimesulida $100 \mathrm{mg}$, de $12 \mathrm{em} 12$ horas, por 72 horas, paracetamol $750 \mathrm{mg}$, de 6 em 6 horas, por 48 horas, e cefadroxil $500 \mathrm{mg}$, de $12 \mathrm{em} 12$ horas, por 7 dias. A paciente foi orientada a registrar de forma escrita caso fosse necessário o uso de qualquer medicação além das prescritas para controle da dor.

Os parâmetros examinados na paciente incluíram dor, número de analgésicos ingeridos, trismo e edema de face. A paciente foi avaliada no pré-operatório e no pós-operatório, nos primeiros três dias e no sétimo dia após o procedimento. Todos os exames de controle pós-operatório e mensurações faciais foram realizados pelo mesmo operador, seguindo o mesmo horário, e as cirurgias também foram feitas pelo mesmo cirurgião.

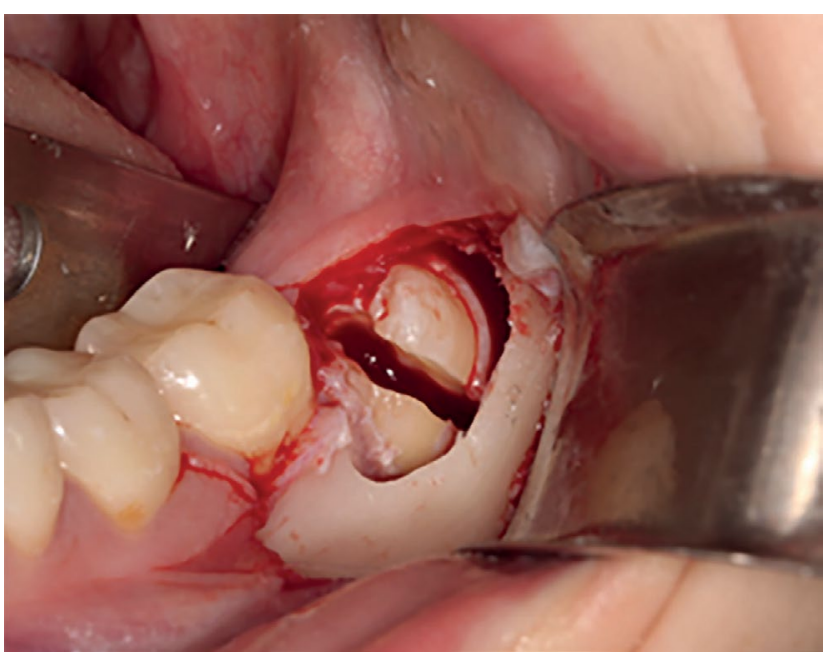

Figura 14 - Odontosecção com broca H33L

Fonte: dos autores.

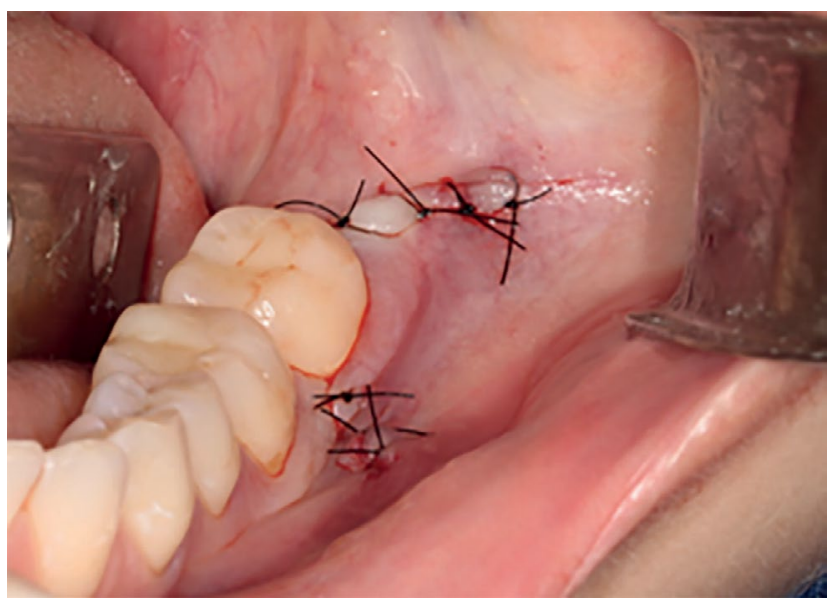

Figura 16 - Sutura da região do 38

Fonte: dos autores.

\section{Análise dos dados}

Os dados clínicos foram avaliados durante cada retorno do paciente, nos primeiros três dias e no sétimo dia do pós-operatório, e as mensurações realizadas foram computadas em uma tabela de dados para cada parâmetro avaliado bilateralmente. Não foi realizada estatística por se tratar de um único relato de caso.

\section{Questões éticas}

$\mathrm{O}$ paciente participante da pesquisa assinou o termo de consentimento livre e esclarecido (TCLE), após a aprovação da pesquisa pelo Comitê de Ética em Pesquisa (CEP) da Imed, pelo parecer de número 1.055.198. 


\section{Resulltados}

A pesquisa incluiu um paciente como estudo-piloto, o qual foi avaliado no pré-operatório quanto a abertura de boca e edema. No pós-operatório, no mesmo dia, foi avaliada a queixa principal descrita pelo paciente e a quantidade de analgésicos ingerida. Todos os parâmetros foram avaliados nos primeiros três dias e no sétimo dia após a cirurgia, junto com a aplicação da escala analógica visual (VAS). O estudo também verificou o tempo cirúrgico de cada técnica utilizada.

O primeiro critério a ser aferido antes das extrações dos terceiros molares foi a abertura de boca, utilizando régua milimetrada flexível, determinando a distância entre os bordos incisais dos incisivos centrais superiores e inferiores; por consequência, a aferição dessa medida não foi bilateral, mensurando o lado direito separadamente do lado esquerdo. A abertura de boca inicial foi de $37 \mathrm{~mm}$, já nos primeiros três dias do pós-operatório, obteve-se, respectivamente, $24 \mathrm{~mm}, 27 \mathrm{~mm}$ e $28 \mathrm{~mm}$. No sétimo dia, a abertura foi medida em $32 \mathrm{~mm}$.

O edema, tanto no pré como no pós-operatório, foi mensurado por meio de um fio de linho e transferido em centímetros para uma régua reta, medindo em 2 pontos, dos dois lados da face, como referência: da comissura labial ao tragus auditivo e da comissura labial ao lóbulo inferior. A medida inicial do lado direito da comissura labial ao alto tragus foi de $11,4 \mathrm{~cm}$, e até ao lóbulo inferior, $10,8 \mathrm{~cm}$. Do lado esquerdo, a primeira medida foi de $11,6 \mathrm{~cm}$, e a segunda, 10,7 cm. Nos outros dias do pós-operatório, foi mensurado apenas da comissura labial ao alto tragus, que obteve, do lado direito, as medidas de: $11,4 \mathrm{~cm}, 1^{\circ}$ dia; $11,7 \mathrm{~cm}, 2^{\circ}$ e $3^{\circ}$ dias; e $11,8 \mathrm{~cm}, 7^{\circ}$ dia. O lado esquerdo foi verificado no $1^{\circ}$ dia, com $11,9 \mathrm{~cm}$, no $2^{\circ}$ dia, $12,2 \mathrm{~cm}$, e no $3^{\circ}, 11,7 \mathrm{~cm}$, continuando com $11,7 \mathrm{~cm}$, no $7^{\circ}$ dia.

Em relação ao tempo cirúrgico, constatou-se a duração média utilizando o dispositivo piezoelétrico em associação com a fibrina rica em plaquetas (PRF): do lado direito, foi de 50 minutos, e do lado esquerdo, com instrumento rotatório convencional e coágulo, foi a metade, em média, 25 minutos. A associação do piezoelétrico com o PRF foi de importância significativa para a aceleração do reparo da ferida, comparando a cicatrização com o lado oposto.

O controle da dor foi avaliado pela escala analógica visual (Visual Analogue Scale - VAS), menos no pós-operatório do mesmo dia. A classificação da escala de VAS é marcada de 0 a 10 , em que 0 indica "ausência de dor" e 10, "dor intensa". No $1^{\circ}$ e no $2^{\circ}$ dia, a dor foi indicada na escala como 1 e, no $3^{\circ}$ dia, como 0 . A partir do $4^{\circ}$ até o $7^{\circ}$ dia, a dor foi apontada entre 4 e 5 (dor moderada), pois o paciente relatou como queixa principal, no $4^{\circ}$ dia: "pus e gosto ruim na boca", devido a traumatismo de tecido mole. A contar do $5^{\mathrm{o}}$ dia, foi relatado dor latejante na articulação temporomandibular, alterando a medicação padrão, para um analgésico a mais por dia. A medicação nos dias anteriores foi a de rotina, prescrita no pós-operatório. $O$ paciente não apresentava qualquer tipo de alteração na articulação temporomandibular anteriormente à cirurgia.

As queixas nos dias anteriores foram relatadas pelo paciente como uma "área latejante" do lado direito, após passar o efeito da anestesia, o que é constatado como normal após a cirurgia. No terceiro dia, como "enjoo ao tomar os remédios pela manhã", pela própria medicação, visto que esse sintoma é uma reação adversa que pode ocorrer quando ministrado algum tipo de antibiótico.

Quadro 1 - Avaliação dos parâmetros propostos no transoperatório e no pós-operatório

\begin{tabular}{|c|c|c|c|c|c|}
\hline & 05/jul. & 06/jul. & 07/jul. & 08/jul. & 12/jul. \\
\hline Abertura de boca & $37 \mathrm{~mm}$ & $24 \mathrm{~mm}$ & $27 \mathrm{~mm}$ & $28 \mathrm{~mm}$ & $32 \mathrm{~mm}$ \\
\hline $\begin{array}{l}\text { Comissura - tragus } \\
\text { Auditivo (edema) }\end{array}$ & $\begin{array}{l}\text { Direito: } 11,4 \mathrm{~cm} \\
\text { Esquerdo: } 11,9 \mathrm{~cm}\end{array}$ & $\begin{array}{l}\text { Direito: } 11,4 \mathrm{~cm} \\
\text { Esquerdo: } 11,9 \mathrm{~cm}\end{array}$ & $\begin{array}{l}\text { Direito: } 11,7 \mathrm{~cm} \\
\text { Esquerdo: } 12,2 \mathrm{~cm}\end{array}$ & $\begin{array}{l}\text { Direito: } 11,7 \mathrm{~cm} \\
\text { Esquerdo: } 11,7 \mathrm{~cm}\end{array}$ & $\begin{array}{l}\text { Direito: } 11,8 \mathrm{~cm} \\
\text { Esquerdo: } 11,7 \mathrm{~cm}\end{array}$ \\
\hline $\begin{array}{l}\text { Comissura - lóbulo } \\
\text { inferior (edema) }\end{array}$ & $\begin{array}{l}\text { Direito: } 10,8 \mathrm{~cm} \\
\text { Esquerdo: } 10,7 \mathrm{~cm}\end{array}$ & & & & \\
\hline Dor & & 1 & 1 & 0 & 4 \\
\hline Medicação & Rotina & Rotina & Rotina & Rotina & $\begin{array}{l}1 \text { analgésico } \\
\text { a mais }\end{array}$ \\
\hline Queixa principal & $\begin{array}{l}\text { Sensação latejante do lado } \\
\text { direito, após passar o efeito } \\
\text { da anestesia. }\end{array}$ & $\begin{array}{l}\text { Ausência de } \\
\text { parestesia. }\end{array}$ & $\begin{array}{l}\text { Enjoo ao tomar a } \\
\text { medicação pela } \\
\text { manhã. }\end{array}$ & $\begin{array}{l}\text { Ausência de } \\
\text { sintomas. }\end{array}$ & $\begin{array}{l}\text { Dor latejante } \\
\text { na articulação } \\
\text { temporomandibular. }\end{array}$ \\
\hline Tempo cirúrgico & $\begin{array}{l}\text { Piezocirúrgico: } 50 \text { minutos } \\
\text { Convencional: } 25 \text { minutos }\end{array}$ & & & & \\
\hline
\end{tabular}

Fonte: dos autores. 
Quadro 2 - Avaliação do pós-operatório registrado pelo paciente no $4^{o}, 5^{o}$ e $6^{o}$ dias

\begin{tabular}{|l|l|l|l|}
\cline { 2 - 4 } \multicolumn{1}{c|}{} & \multicolumn{1}{c|}{ 09/jul. } & \multicolumn{1}{c|}{$10 /$ jul. } & \multicolumn{1}{c|}{$11 /$ jul. } \\
\hline Dor & 4 & 5 & 5 \\
\hline Medicação & Rotina & 1 analgésico a mais & 1 analgésico a mais \\
\hline $\begin{array}{l}\text { Queixa } \\
\text { principal }\end{array}$ & $\begin{array}{l}\text { Relato de pus, } \\
\text { e gosto ruim. }\end{array}$ & $\begin{array}{l}\text { Dor latejante na } \\
\text { articulação } \\
\text { temporomandibular. }\end{array}$ & $\begin{array}{l}\text { Dor latejante na } \\
\text { articulação } \\
\text { temporomandibular. }\end{array}$ \\
\hline
\end{tabular}

Fonte: dos autores.

\section{Discussão}

Nesse estudo, ambas as técnicas para osteotomia em terceiro molar inferior impactado, a piezocirurgia em associação com o PRF e a utilização de instrumentos rotatórios convencionais e coágulo, feitas em lados opostos, foram executadas no mesmo dia, de maneira simultânea, justamente para que fosse realizado o mesmo protocolo cirúrgico e o mesmo protocolo terapêutico medicamentoso, para normatizar os resultados dos parâmetros abordados, como edema e indicação de dor pela escala VAS.

As técnicas de osteotomia foram realizadas no mesmo paciente, como estudo-piloto, para que não houvesse, pelo mesmo motivo, discrepância dos resultados, já que alguns autores trazem pesquisas feitas em dois grupos de pacientes para a comparação do piezoelétrico com a osteotomia convencional ${ }^{8-9}$.

Em exodontia de terceiros molares impactados, a limitação da abertura de boca, pode ser causada por edema no pós-operatório, sendo uma causa extra-articular, relacionada a diversos fatores, como a dificuldade do procedimento cirúrgico, o que envolve a severidade da retensão e a técnica utilizada, consequentemente, pelo tempo durante o transoperatório ${ }^{10-11}$.

Na pesquisa, observou-se que o tempo cirúrgico do piezoelétrico foi o dobro que o do instrumento rotatório convencional, o que deveria, desse modo, trazer maior limitação de abertura de boca, como mencionado anteriormente, porém, a medida mais relevante foi no $1^{\circ}$ dia do pós-operatório, mensurado em $24 \mathrm{~mm}$. No $7^{\circ}$ dia, a abertura já correspondia a $32 \mathrm{~mm}$, uma diferença de $5 \mathrm{~mm}$ em relação à medida inicial.

Um estudo realizado em 2012 demonstrou que o grupo controle (utilizando piezoelétrico) obteve limitação da abertura de boca maior em comparação ao convencional e que os valores foram semelhantes após 15 dias. Entretanto, outros critérios, como dor e número de analgésicos ingeridos, foram menores nesse grupo, concluindo que, apesar disso, o piezo é uma técnica relativamente mais favorável ${ }^{12}$.

Outro estudo realizado em $2005^{13}$, apenas com instrumento rotatório convencional, verificou que $17,5 \%$ dos pacientes apresentaram a distância in- terincisal (DI) igual ou superior a inicial e, devido a complicações, como processo inflamatório e/ou infeccioso, $7,5 \%$ dos pacientes apresentaram a DI superior se comparado ao pré-operatório. Para Oliveira et al. $^{2}$ (2006), o trismo foi a limitação mais encontrada, principalmente, em casos em que a exodontia foi mais traumática. Como citado, a limitação da abertura bucal será relativa à dificuldade do procedimento em questão e a fatores biológicos do próprio paciente, sendo inevitável em alguns casos, independente da técnica utilizada. Porém, no projeto-piloto, devido ao fato de a cirurgia ser realizada no mesmo ato cirúrgico, essa medida pode ter sido um viés para avaliar o trismo pós-operatório.

Posteriormente a exodontias, o organismo procede como um mecanismo de defesa no local, especialmente pela lesão que é causada ao tecido mole e ao osso, como em extração de dentes inclusos, iniciando o processo inflamatório, gerando dor, calor, rubor e edema. Conforme descrito na literatu$\mathrm{ra}^{14}$, o edema é definido como acúmulo de líquido no espaço intersticial, podendo ser de pequena ou ampla dimensão, dependendo do porte cirúrgico. Assim como mostra os resultados do presente estudo, o edema teve o seu pico máximo no $2^{\circ}$ dia do pós-operatório, no qual foi mensurado $11,7 \mathrm{~cm}$ no lado do piezoelétrico, e $12,2 \mathrm{~cm}$ no lado do método convencional, após passada essa fase, teve-se efeito reverso, regredindo o edema, sendo semelhantes as medidas nos dias seguintes do pós-operatório, até o $7^{\circ}$ dia. Percebemos a diferença em relação ao inchaço, pois o piezoelétrico proporciona um corte mais preciso, seguro e eficaz em comparação ao convencional, além de não lesionar tecidos moles e estruturas anatômicas importantes, tornando-se mais eficiente nas primeiras fases da cicatrização óssea, induzindo um aumento de proteínas morfogenéticas ósseas (BMPs) e controlando a inflamação, diminuindo, assim, o edema ${ }^{15}$.

Um estudo realizado em 2013 demonstrou que a utilização da fibrina rica em plaquetas (PRF) justifica-se pela sua capacidade de proteção dos fatores de crescimento da proteólise, aumentando, dessa forma, seu período de atividade, estimulando de forma mais eficaz a regeneração óssea. Por conseguinte, o nosso estudo demonstrou que a associação do PRF com o piezoelétrico, comparando com a combinação de coágulo e a técnica de osteotomia convencional, teve maior aceleração do reparo da ferida, pois uma das particularidades do PRF é ser um regulador imune com habilidades inflamatórias, explicando a redução de infecções pós-operatórias, quando usada em procedimentos cirúrgicos ${ }^{7,16}$. Logo, a utilização do PRF também contribuiu para que houvesse um controle do processo inflamatório e, posteriormente, do edema. Alguns autores concluíram, em sua pesquisa, que a utilização de PRF combinada com o piezo reduziu significativamente a dor, o número de analgésicos ingeridos e o trismo 24 horas após a 
cirurgia, tendo efeito positivo sobre o desconforto do pós-operatório do paciente ${ }^{17}$.

O controle de dor, em nossa pesquisa, denotou que não se teve dor severa no pós-operatório, a dor máxima foi indicada como 5 (moderada), do $5^{\mathrm{o}}$ ao $6^{\mathrm{o}}$ dia, tendo como queixa principal do paciente uma "dor latejante" na articulação-temporomandibular, evidenciando que a dor local após cirurgia é normal e deve ser controlada com analgésicos, regredindo de forma espontânea, pois normalmente é causada pelo inchaço e em consequência do trismo. Em alguns casos, é necessário prescrever também relaxantes musculares ou até recomendar fisioterapia, no nosso caso clínico, o paciente apenas fez uso de um analgésico a mais por dia, quando o desconforto e a dor foram maiores, até o $7^{\circ}$ dia do pós-operatório, quando a indicação da dor na escala passou a ser 4, regredindo posteriormente.

Estudo realizado em 2015 relatou que o uso do piezoelétrico combinado com o PRF diminuiu significativamente o número de analgésicos ingeridos no trabalho ${ }^{17}$. Em contrapartida, autores que realizaram trabalho em 2015 demonstraram que todos os pacientes avaliados no estudo apresentaram dor, entretanto, confirmaram que o limiar de dor das pessoas é diferente, pois pacientes mais velhos podem ser menos sensíveis a dor, principalmente, nos dois primeiros dias do pós-operatório, por terem mais experiência em relação à dor do que pacientes mais jovens, em que a ingestão de analgésicos após extrações é maior, concluindo que a dor pode estar relacionada também com a experiência pessoal ${ }^{18}$.

Quanto ao tempo cirúrgico, é evidente que o piezoelétrico é uma técnica que necessita de mais tempo se comparado à peça de mão reta e brocas, pois é reduzida a vibração e a pressão sobre o osso. Em um trabalho realizado em 2013, os autores apontaram que o tempo médio levado em cirurgia feita com o método convencional levou 32,73 minutos, e com o piezoelétrico 54,50 minutos, isso vai ao encontro de que nossa pesquisa, que teve um tempo estimado de 25 minutos e 50 minutos, respectivamente ${ }^{19}$. Em contrapartida, uma das principais vantagens do piezoelétrico é o spray de soro fisiológico, que auxilia para resfriar e irrigar a região da incisão, impedindo até o risco de necrose óssea por aquecimento, ao contrário do convencional, que é utilizado o soro e é dependente de auxiliar, que talvez não consiga fazer essa refrigeração de forma adequada.

Como se averiguou, é de extrema importância que esse tipo de exodontia seja indicado em época adequada e, principalmente, que seja avaliado se poderá trazer algum prejuízo futuro ao paciente. Normalmente, essas exodontias são realizadas em pessoas mais jovens, as quais não possuíram a experiência com cirurgias desse tipo, podendo causar transtornos a esses pacientes, como aponta um estudo publicado sobre qualidade de vida após extração de terceiros molares, que 83,3\% dos homens e 59,5\% das mulheres submetidos a esses procedimentos isolam-se por motivos de dor e desconforto no pós-operatório ${ }^{4}$. Dessa maneira, o cirurgião-dentista pode minimizar esses danos, trazendo novas tecnologias menos traumáticas, como o piezoelétrico em associação com o PRF, a fim de reduzir acidentes no transoperatório e complicações no pós-operatório.

\section{Conclusão}

A partir dos resultados obtidos pelo presente estudo, é possível sugerir que a utilização do piezoelétrico associado com o uso do PRF, mesmo que seja prolongado o tempo cirúrgico, pois proporciona um pós-operatório mais favorável em relação ao edema e à dor pós-operatória, já que o piezoelétrico proporciona corte ósseo mais preciso e menos agressivo aos tecidos moles, enquanto que o PRF possui citocinas que são liberadas durante o processo de reparo, estimulando o processo de cicatrização. Por se tratar de um estudo-piloto em que apenas um paciente foi avaliado, sugere-se que mais estudos sejam realizados.

\section{Abstract}

Objective: to evaluate the postoperative results in surgeries of impacted lower third molars with split mouth design. One side received platelet-rich fibrin (PRF) and piezo surgical unit, while the opposite side received conventional rotational osteotomy and clot. We evaluated the relationship between the two osteotomy techniques, surgical time, severity of postoperative sequelae including pain, edema, and trismus, and repair. Case report: this study was performed by means of a clinical case report as the pilot project from a series of clinical cases. The lower third molars indicated for extraction were paired by the Pell and Gregory classification and divided into two groups: Group A (right hemiarch) used the piezo surgical unit and PRF; Group B (left hemiarch) followed the conventional standards with rotating drills and clot. Extraction was performed at a single moment by the same dental surgeon. Piezosurgery compared to the conventional technique showed lower postoperative pain in the Visual Analog Scale and reduced the number of analgesics taken, significantly reducing trismus after 72 hours of surgery. In contrast, the surgical time was superior to the conventional technique. Final considerations: this study concluded that the use of piezo surgical unit and PRF showed better results in the postoperative conditions concerning pain, edema, and repair. However, the surgical time was longer than conventional osteotomy and clot. Therefore, the present study shows that piezosurgery and the combined use of PRF have positive effects on the postoperative results after impacted third molar extraction.

Keywords: Osteotomy. Minor surgery. Third molar. Extraction. 


\section{Referências}

1. Aquino B, Vieira P. Exodontia em terceiro molar inferior incluso: complicações e acidentes. IV Encontro de iniciação científica da Unicor, 2012; Belo Horizonte MG. Anais. Revista de iniciação científica da Universidade Vale do Rio Verde; 2012. p. 23

2. Oliveira L, Schmidt D, Assis A, GabrielIi M, Vieira E, Pereira V. Avaliação dos acidentes e complicações associados à exodontias dos $3^{\circ}$ molares. Rev Cir Traumatol Buco-Maxilo-Fac 2006; 6(2):51-6.

3. Araújo O, Agostinho C, Marinho L, Rabêlo L, Bastos E, Silva V. Incidência dos acidentes e complicações em cirurgias de terceiros molares. Rev Odontol Unesp 2011; 40(6):290-5.

4. Santos T, Santos E, Lins R, Araújo L, Mesquita B, Sobreira T. Qualidade de vida de pacientes submetidos à exodontia de terceiros molares. Rev Odontol Unesp 2015; 44(1):6-11.

5. Stubinger S, Kuttenberger J, Filippi A, Sader R, Zeilhofer F. Intraoral piezosurgery: Preliminary results of a new technique. J Oral Maxillofac Surg 2005; 63:1283-7.

6. Watanabe E, Gabrielli M, Vieira E, Filho R, Freitas O. Utilização do motor piezoelétrico em cirurgia buco maxilo facial. Rev Odontol Unesp 2013; 42(Especial):141.

7. Dohan DM, Choukroun J, Diss A, Dohan SL, Mouhyi J, Gogle B. Platelet-rich fibrin (PRF): a second-generation platelet concentrate. Part I: Technological concepts and evolution. Oral Surg Oral Med Oral Pathol Oral Radiol Endod 2006; 101:E37-44.

8. Piersanti L, Dilorenzo M, Monaco G, Marchetti C. Piezosurgery or conventional rotatory instruments for inferior third molar extractions? J Oral Maxillofac Surg 2014; 72:1647-52.

9. Jiang Q, Qiu Y, Yang C, Yang J, Chen M, Zhang Z. Piezoelectric versus conventional rotary techniques for impacted third molar extraction: a meta-analysis of randomized controlled trials. Medicine (Baltimore) 2015; 94(41):e1685.

10. Sortino F, Pedulla E, Masoli V. The piezoelectric and rotatory osteotomy technique in impacted third molar surgery: comparison of postoperative recovery. J Oral Maxillofac Surg 2008; 66(12):2444-8.

11. Mantovani E, Arduino PG, Schierano G, Ferrero L, Gallesio G, Mozzati M, et al. A split-mouth randomized clinical trial to evaluate the performance of piezosurgery compared with traditional technique in lower wisdom tooth removal. J Oral Maxillofac Surg 2014; 72(10):1890-7.

12. Goyal M, Marya K, Jhamb A, Chawla S, Sonoo PR, Singh $\mathrm{V}$, et al. Comparative evaluation of surgical outcome after removal of impacted mandibular third molars using a Piezotome or a conventional handpiece: a prospective study. $\mathrm{Br} \mathrm{J}$ Oral Maxillofac Surg 2012; 50:556-61.

13. Aguiar A, Oliveira A, Martins P, Freire R. Avaliação do grau de abertura bucal e dor pósoperatória após a remoção de terceiros molares inferiores retidos. Rev Cir Traumatol Buco-Maxilo-Fac 2005; 5(3)57-64.

14. Coelho E. Mecanismos de formação de edema. Medicina Ribeirão Preto 2004; 37:189-98.

15. Labanca M, Azzola F, Vinci R, Rodella L. Piezoelectric surgery: twenty years of use. Br J Oral Maxillofac Surg 2008; 46:265-9.

16. Mallmann F, Lago P, Bona A. Uso de fibrina rica em plaquetas (PRF) no tratamento de perfurações da membrana sinusal. Full Dent Sci 2013; 5(7):59-66

17. Uyanik L, Bilgnaylar K, Etikan I. Effects of platelet-rich fibrin and piezosurgery on impacted mandibular third molar surgery outcomes. Head \& Face Medicine 2015; 11(25):1-7.
18. Tasveer F, Gupta H, Kumar D. Peizoelectric Ostectomy: a new technique for impacted third molar surgery. J Med Dent Sci 2015; 14(9):103-7.

19. Bartuli F, Luciani F, Caddeo F, Chiara L, Dio L, Piva P, et al. Piezosurgery vs high speed rotary handpiece: a comparasion between the two tchniques in the impacted third molar surgery. Oral Implantol 2013; 6(1):5-10.

\section{Endereço para correspondência:}

Gabriel Rodrigues Oliveira

Rua Bento Gonçalves, 50, sala 401, Centro CEP 99010-010, Passo Fundo, RS, Brasil

Telefone: (54) 33135042

E-mail: grodriguesoliveira2@yahoo.com.br vinifabris@hotmail.com

Recebido: 29/06/2017. Aceito: 29/07/2017. 


\title{
Apêndice A
}

\author{
TERMO DE CONSENTIMENTO LIVRE E ESCLARECIDO
}

Prezada Sra. Natália Pittol, estamos desenvolvendo um estudo que visa verificar se a fibrina rica em plaquetas quando associado a utilizaçăo de piezzo cirúrgico quando comparado a técnica cirúrgica convencional de exodontia de terceiros molares é capaz de proporcionar uma cicatrizaçăo e um pós operatório de uma forma mais rápida eficiente, cujo título é Fibrina rica em plaquetas e piezocirúrgico em comparação com instrumento rotatório convencional e coágulo em cirurgia de terceiros molares inferiores impactados: relato de caso. Você está sendo convidado a participar deste estudo.

Esclareço que durante $\circ$ trabalho năo haverá riscos ou desconfortos, nem tampouco custos ou forma de pagamento pela sua participaçăo no estudo.

Eu. Vinicius Fabris e a minha equipe e Gabriel Rodrigues Oliveira, estaremos sempre à disposiçăo para qualquer esclarecimento acerca dos assuntos relacionados ao estudo, no momento em que desejar, através do telefone 5491585807 e do endereço Rua Bento Gonçalves n` 50 sala 401 cep 99010-010.

É importante que você saiba que a sua participaçăo neste estudo é voluntária e que você pode recusar-se a participar ou interromper a sua participaçăo a qualquer momento sem penalidades ou perda de beneficios aos quais vocé tem direito.

Pedimos a sua assinatura neste consentimento. para confirmar a sua compreensão em relação a este convite. e sua disposiçăo a contribuir na realizaçăo deste trabalho, em concordância com a Resoluçăo CNS n 196/96 que regulamenta a realização de pesquisas envolvendo seres humanos.

Desde já agradecemos a sua atençăo.

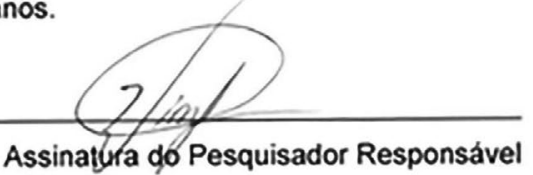

Eu, Notólia Rittol após a leitura deste consentimento, declaro que compreendi o objetivo deste estudo e confirmo o meu interesse em participar desta pesquisa.

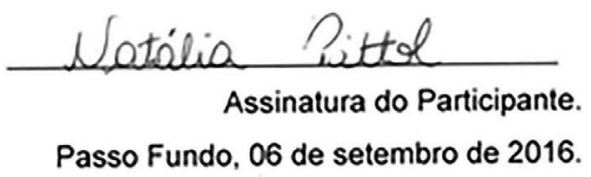




\section{Apêndice B}

FACULDADE MERIDIONAL IMED/RS

\section{PARECER CONSUBSTANCIADO DO CEP}

\section{DADOS DO PROUETO DE PESQUISA}

Titulo da Pesquisa: ESTUDO COM DESENHO SPUT MOUTH DE RECONSTRUCĀO ÓSSEA DE MAXILA ATRÓFICA ASSOCLADA AO USO DE PRF: ANÁLISE CLINICA E HISTOLÓGICA

Pesquisador: VINICIUS FABRIS

Ärea Temítica:

Versāo: 2

CAAE: 43143014.6 .0000 .5319

Instituiçäo Proponente: Faculdade Meridional - IMED

Patrocinador Principal: Financiamento Próprio

DADOS DO PARECER

Nümero do Parecer: 1.055 .198

Data da Relatoria: 06/05/2015

Apresentagiāo do Projeto:

Trata-se de estudo sobre pacientes que [-.] apresentam quadro de atrofia maxilar severa, onde a condicäo óssea é minima devido a reabsorçāo óssea cumulativa ao longo dos anos, esse quadro dificulta o tratamento convencional com implantes dentärios, impossibilitando a reabilitaçäo oral do paciente. 0 reestabelecimento da funçāo e também a devoluçäo do suporte dos tecidos da face que foram perdidos em decorrència do edentulismo. Uma das formas para o tratamento desses casos. onde a reabilitaçäo com implantes convencionais näo è possivel, é atravès de enxertos ósseos e reconstruçöes com biomateriais. sendo que atualmente. para melhorar o processo de reparo ósseo a fibrina rica em plaquetas (PRF) vem sendo associada aos enxertos com o objetivo de acelerar o processo de cicatrizaçāo".

Objetivo da Pesquisa:

-Analisar a influència do uso da fibrina rica em plaquetas sobre o processo de regeneraçāo óssea quando comparada a técnica cirúrgica convencional de enxerto em bloco e levantamento de seio bilateral com a utiliczaçäo de osso autögeno".

Avaliagäo dos Riscos e Beneficios:

Riscos inerentes ao procedimento cińrgico como: edema, sangramento, hemorragia, infeç̧äo de ärea doadora e receptora, perda de implantes, atraso na finalizaçäo da reabilitação em caso de 